\title{
Community Urinary Tract Infection due to ESBL producing $E$. coli: epidemiology and susceptibility to oral antimicrobials including Mecillinam
}

\author{
Datta P, $^{1}$ Gupta $V^{1}$ Sidhu $S,{ }^{1 *}$ Chander $J^{1}$ \\ ${ }^{1}$ Department of Microbiology, Government Medical College Hospital, Chandigarh, India
}

\author{
*Corresponding Author: \\ Dr. Shailpreet Sidhu \\ Senior Resident, \\ Department of Microbiology, \\ Government Medical College Hospital, \\ Sector 32, Chandigarh (India)-160030 \\ Email: shailpreet78@hotmail.com
}

\section{Citation}

Datta P, Gupta V, Sidhu S, Chander J. Community Urinary Tract Infection due to ESBL producing E. coli: epidemiology and susceptibility to oral antimicrobials including Mecillinam. Nepal Journal of Medical sciences 2014;3(1):5-7.

\section{Background:}

Community acquired urinary tract infection (UTI) due to Escherichia coli is one of the most common form of bacterial infections, affecting people of all ages. Originally ESBL (Extended Spectrum $\beta$-lactamases) producing $E$. coli was isolated from hospital setting but lately this organism has begun to disseminate in the community. ${ }^{1}$

\begin{abstract}
Background: There is increasing incidence of (Extended spectrum beta lactamases) ESBL producing E. coli causing community urinary tract infections (UTI). The primary objective of this study was to study the epidemiological factors associated with ESBL positive community acquired uropathogenic $E$. coli isolates and to determine their susceptibility to newer oral drugs including mecillinam.

Methods: In this prospective study, from total of 140 community isolates of E. coli causing UTI, ESBL was detected by Clinical Laboratory Standards Institute criteria. Drug susceptibility was done by Kirby-Bauer method disc diffusion method for various oral antimicrobial agents. Various epidemiological factors associated with ESBL for each patient were recorded on individual forms. This included age, presence of diabetes mellitus, renal calculi, pregnancy, history of urinary instrumentation, recurrent UTI and antibiotics intake.
\end{abstract}

Results: Out of total of 140 strains of $E$. coli, which were screened for ESBL production, 30 (21.4\%) isolates were positive. High-level resistance $94(70 \%)$ was seen for many antimicrobial agents. Only $4.5 \%$ of uropathogenic E. coli were resistant to Mecillinam. Various epidemiological factors associated with ESBL causing infections were female patients, $\mathrm{H} / \mathrm{o}$ antimicrobial intake, elderly age $>60$ years, renal calculi and $\mathrm{H} / \mathrm{o}$ recurrent UTI.

Conclusions: The epidemiology of ESBL positive uropathogenic E. coli is becoming more multifaceted.

Keywords: Community UTI; E.coli; epidemiology; ESBL 
patients with UTI warrants a thorough knowledge of local epidemiology and patient's profile. Therefore, the primary objective of this study was to determine the epidemiological factors associated with ESBL positive community acquired uropathogenic $E$. coli isolates and to determine their susceptibility to newer oral drugs. Mecillinam is a novel $\beta$-lactam antibiotics that is active against many Enterobacteriaceae. It binds to PBP2, an enzyme critical for the establishment and maintenance of bacillary cell shape. It is given as a prodrug that is hydrolyzed into active agent. It is well tolerated orally in the treatment of acute cystitis. ${ }^{3}$

\section{Methods:}

This prospective study was conducted in Government Medical College Hospital, Chandigarh, India, which caters to medical needs of the community from North India from Jan 2012- July 2012.

\section{Study group}

It included patients diagnosed as having UTI in outpatient clinic or emergency room or patients diagnosed within $48 \mathrm{hrs}$ after hospitalization. These patients were labeled as having community UTI. A diagnosis of symptomatic UTI was made when patient had at least one of the following signs or symptoms with no other recognized cause: fever $\geq 38.8^{\circ} \mathrm{C}$, urgency, frequency, dysuria or suprapubic tenderness and patient had a positive urine culture i.e. $\geq 10^{5}$ microorganisms/ $\mathrm{ml}$ of urine. ${ }^{4}$ Various epidemiological factors for each patient were recorded on individual forms. This included age, presence of diabetes mellitus, renal calculi, pregnancy, history of urinary instrumentation, recurrent UTI (more than 3 UTI episodes in the preceding year) and antibiotics intake (use of $\beta$-lactam in the preceding 3 months). ${ }^{2}$

Patients with $\mathrm{H} / \mathrm{O}$ previous or recent hospitalizations were excluded from study.

Antibiotic susceptibility testing was carried out following Clinical Laboratory Standards Institute (CLSI) guidelines using Kirby-Bauer method. ${ }^{5}$ The antibiotics which were tested included amoxyclav $(30 / 10 \mu \mathrm{g})$, norfloxacin $(10 \mu \mathrm{g})$, ciprofloxacin $(5 \mu \mathrm{g})$, tetracycline $(30 \mu \mathrm{g})$, nitrofurantoin $(300 \mu \mathrm{g})$, trimethoprim-sulfamethoxazole $(23.75 / 1.25 \mu \mathrm{g})$, cephalexin $(30 \mu \mathrm{g})$, cefaclor $(30 \mu \mathrm{g})$, Cefuroxime $(30 \mu \mathrm{g})$, Mecillinam $(10 \mu \mathrm{g})$ (Hi-Media, Mumbai, India)

\section{Detection of ESBL}

ESBL detection was done for all isolates according to latest CLSI criteria. $^{5}$

Screening test- According to latest CLSI guidelines, zone diameter of E. coli strain for ceftazidime $<22 \mathrm{~mm}$ and for cefotaxime $<21 \mathrm{~mm}$ is presumptively taken to indicate ESBL production.

Confirmatory test- As per CLSI guidelines, ESBLs were confirmed by placing disc of cefotaxime and ceftazidime at a distance of $20 \mathrm{~mm}$ from a disc of cefotaxime /clavulanic acid $(30 / 10 \mu \mathrm{g})$ and ceftazidime/ clavulanic acid $(30 / 10 \mu \mathrm{g})$ respectively on a lawn culture of test strain ( $0.5 \mathrm{McF}$ arland inoculum size) on Mueller-Hinton agar. After overnight incubation at $37^{\circ} \mathrm{C}, \mathrm{ESBL}$ production was confirmed if there $\geq 5 \mathrm{~mm}$ increase in zone diameter for either antimicrobial agent tested in combination with clavulanic acid versus its zone when tested alone.

Control strain - Standard strain of Klebsiella pneumonia ATCC 700603 was used as ESBL positive control and Escherichia coli ATCC 25922 was used as ESBL negative control.

\section{Results:}

Out of total of 140 strains of $E$. coli, which were screened for ESBL production, 30 (21.4\%) isolates were positive. Highlevel resistance was seen for many antimicrobial agents like cephalexin $(92.8 \%)$, cefaclor $(90 \%)$, amoxy-clavulanate $(88.57 \%)$, cefuroxime $(75.7 \%)$, sulfamethoxazoletrimethoprim (72.8\%), norfloxacin $(75.71 \%)$ and ciprofloxacin $(70 \%)$. Whereas, sensitivity for nitrofurantoin was high (90\%). Only $4.5 \%$ of uropathogenic E. coli were resistant to Mecillinam.

Various epidemiological factors seen in ESBL producers include female patients ( $\mathrm{n}=24,80 \%), \mathrm{H} / \mathrm{o}$ antimicrobial intake $(n=17,57 \%)$, elderly age $>60$ years $(n=1653 \%)$, renal calculi $(\mathrm{n}=15,50 \%), \mathrm{H} / \mathrm{o}$ recurrent UTI $(\mathrm{n}=11,37 \%)$, pregnancy $(n=11,37 \%)$, diabetes mellitus $(n=7,23 \%)$ and $\mathrm{H} / \mathrm{O}$ urogenital instrumentation $(\mathrm{n}=7,23 \%)$.

\section{Discussion:}

The epidemiology of ESBL positive uropathogenic E. coli is becoming more multifaceted, with increasingly indistinct boundaries between the community and hospital [6]. In addition, infection with an ESBL producing organisms causing community UTI is associated to treatment failure, delayed clinical response, higher morbidity and mortality. These organisms are mutiresistant to other antimicrobials like aminoglycosides, quinolones and co-trimoxazole. Therefore, empirical therapy with cephalosporins and fluoroquinolones often fail in these patients with community UTI. $^{7}$

The rate of ESBL producers in our study are lower than that described by other authors. In a similar study Mahesh E et al reported higher rate $(56.2 \%)$ of ESBL positivity from $E$. coli, which were causing UTI from community setting 
respectively. ${ }^{8}$ Additionally Taneja $\mathrm{N}$ et al described a higher rate $(36.5 \%)$ of ESBL positivity in uropathogens. ${ }^{9,10}$

A high rate of resistance was seen to almost all antimicrobial agents. This is in agreement with other studies. ${ }^{8,11}$ Mecillinam showed very good results with only $4.5 \%$ resistance. Wootton $\mathrm{M}$ et al reported similar high activity of mecillinam against E. coli $(93.5 \%){ }^{12}$ Auer $\mathrm{S}$ et al reported that mecillinam can be a good oral treatment options in patients with infections due to ESBL organisms. ${ }^{7}$

Limitations of our study was that being a developing country with limited resources, molecular typing and determination of antimicrobial resistance profiles of the isolates was not done. Since description of ESBL types may have be of some interest for epidemiological purposes.

In our study female patients, elderly, patients with $\mathrm{H} / \mathrm{o}$ antimicrobial intake, renal calculi and $\mathrm{H} / \mathrm{o}$ recurrent UTI were important epidemiological factors for infections due to ESBL producers. This is similar to risk factors studied by Azap OK et al. In conclusion, the prevalence of ESBL producers causing community UTI is less and mecillinam is a good treatment option.

\section{Conflict of interest: none}

Funding: none

\section{References:}

1. Rodríguez-Baño J, C Alcalá J, M Cisneros J, et al. Community Infections Caused by Extended-Spectrum $\beta$-Lactamase-Producing Escherichia coli. Arch Intern Med 2008;168:1897-902.

\section{http://dx.doi.org/10.1001/archinte.168.17.1897}

2. Azap OK, Arslan H, erefhanog KS, et al. Risk factors for extended-spectrum b-lactamase positivity in uropathogenic Escherichia coli isolated from community-acquired urinary tract infections. Clin Microbiol Infect 2010;16:147-51.

\section{http://dx.doi.org/10.1111/j.1469-0691.2009.02941.x}

3. Wootton M, Walsh TM, Macfarlane L, et 1. Activity of mecillinam against Escherichia coli resistant to thirdgeneration cephalosporins. J Antimicrob Chemother 2010;65:79-81.

\section{http://dx.doi.org/10.1093/jac/dkp404}

4. Lee DS, Lee CB, Lee SJ. Prevalence and Risk Factors for Extended Spectrum Beta-Lactamase-Producing
Uropathogens in Patients with Urinary Tract Infection. Korean J Urology 2010;51:492-7.

\section{http://dx.doi.org/10.4111/kju.2010.51.7.492}

5. Clinical and Laboratory Standards Institute. Performance Standards for Antimicrobial Susceptibility Testing: Twenty First Informational Supplement M100-S21. CLSI, Wayne, PA, USA, 2011.

6. Nesher L, Novack V, Riesenberg F, et al. Regional community-acquired urinary tracts in Israel; diagnosis, pathogens, and antibiotics guidelines adherence: A prospective study. International $\mathrm{J}$ Infect Dis 2007;11:245-50.

\section{http://dx.doi.org/10.1016/j.ijid.2006.03.004}

7. Auer S, Wojna A, Hell M. Oral Treatment Options for Ambulatory Patients with Urinary Tract Infections Caused by Extended-Spectrum beta-LactamaseProducing Escherichia coli. Antimicro Agents Chemo 2010,54:4006-8.

\section{http://dx.doi.org/10.1128/AAC.01760-09}

8. E Mahesh, Ramesh D, Indumathi VA, et al. Risk Factors for Community Acquired Urinary Tract Infection caused by ESBL-producing Bacteria. JIACM 2010;11:271-6.

9. Taneja N, Rao P, Arora J, et al. Occurrence of ESBL \& Amp-C $\beta$-lactamases \& susceptibility to newer antimicrobial agents in complicated UTI. Indian J Med Res 2008;127: 85-8.

10. Taneja N, Singh G, Singh M, et al. High occurrence of blaCMY-1 Amp C lactamase producing Escherichia coli in cases of complicated urinary tract infection (UTI) from a tertiary health care centre in north India. Indian J Med Res 2012;136:289-91.

11. Mandal J, Acharya NS, Buddapriya D, et al .Antibiotic resistance pattern among common bacterial uropathogens with a special reference to ciprofloxacin resistant Escherichia coli. Indian J Med Res 2012;136:842-9.

12. Wootton M, Walsh TM, Macfarlane L, et al. Activity of mecillinam against Escherichia coli resistant to thirdgeneration cephalosporins. J Antimicrob Chemother 2010;65:79-81.

\section{http://dx.doi.org/10.1093/iac/dkp404}

